\title{
Factors Relating to Nurses' End-of-Life Care
}

\author{
Li Xia, Waraporn Kongsuwan \\ Adult and Elderly Nursing Department, Faculty of Nursing, Prince of Songkla University, Hatyai, Songkhla, Thailand \\ Email:xiali748@gmail.com,waraporn.k@psu.ac.th,waraporn_kongsuwan@yahoo.co.uk
}

How to cite this paper: Xia, L. and Kongsuwan, W. (2020) Factors Relating to Nurses' End-of-Life Care. Journal of Biosciences and Medicines, 8, 189-200. https://doi.org/10.4236/jbm.2020.86018

Received: May 27, 2020

Accepted: June 27, 2020

Published: June 30, 2020

\begin{abstract}
This integrative review aimed to investigate factors relating to end-of-life care of nurses. The review was conducted according to PICo (Participant, area of Interest, and Context). Keywords identified were: "nurse" AND "end-of-life care" ("dyingcare" OR "deathcare", OR "near end stage of life care" OR "palliative care" OR "hospice care" OR "comfortable care in near death" OR "quality of dying patients care"), AND "nurses". The database searched through PubMed, ProQuest, Google Scholar, Web of Science and SCOPUS. The searching inclusion criteria were limited to English and Chinese language studies about nurses' end-of-life care from 2010 to 2019, yielding 258 English language articles and 2Chinese language articles. Results: A total of fifteen articles were selected based on inclusion criteria. Two subjects were obtained from the results that related to nurses' care during the process of end of life: 1) Nurses' demographic factors; and 2) Modifiable factors. Nurses' demographic factors were age, years of work experience, level of education and experience of the death of a family member/friend. Modifiable factors included knowledge, attitude, confidence, relationship, environment and resources, communication, nursing activities, philosophy and culture of care, skills and training. These factors were explored in various areas and majority of the studies had been conducted in public government hospitals. Conclusion: The major power ability of care was observed in nurses who acted the significant part in caring for the terminally ill during the dying process. Caring for dying patients was related to many factors which could affect the fabric of nursing care at the end stage of life. The elements found in this review could lead to recommendations with implications for nursing practice so as to improve and enhance end-of-life care. Some factors could be considered like predictors affecting nursing practice for chronical ill patients in further research. In addition, nurses' tranquility care in community hospitals should be more focused.
\end{abstract}

\section{Keywords}

End of life, Care, Dying, Relating Factors, Nurses 


\section{Introduction}

End-of-life care is defined as the overall nursing care provided to patients in an advanced stage of illness with pain control and other symptoms relief, and social, psychological and spiritual care, which can help sick persons and their household get the best quality of livelihood [1]. It can also be applied to some early patients, such as the relief of early pain in cancer patients. A report described that many patients hoped the nurses could care for them well during the final process of their lives [2]. The nurse had to respond to demands of dying patients in each criterion of peaceful care with appropriate caring actions [3].

But severe deficiencies in this domain were observed in most nations, and the demand for end-of-life care is expected to grow greatly owing to demographic development with more and more elderly and cancer patients with chronic diseases beyond cure and multiple health conditions, because most people were usually diagnosed in a later period [4]. As a large country with an aging population, China is facing severe challenges in the treatment and nursing of cancer patients and other geriatric patients. Since the end-of-life care in our country has started not so long ago, there are many issues that need to be explored and studied [5]. End-of-life care of nurses is one of the most significantly essential links, and a keyword in end-of-life care is indeed "care", instead of using the word "treat", emphasizing that nurses will play an increasingly important role and that Chinese nurses will need to learn and improve their professional level. However, at present, the practice content of the end-of-life care in China is not very clear [5]. Therefore, in order to improve nurses' quality of end of life care for our people, understanding and mastering the factors that affect nurses' end-of-life care is a very important step.

\section{Objective}

To review factors related to end-of-life care of nurses from existing documents.

\section{Method}

\subsection{Design}

This study employed an integrative review method which had five steps: 1) Identifying the question: Guaranteeing that the research question and object are defined clearly; 2) Literature search: Combining an all-around search scheme; 3) Data evaluation: Focusing on authenticity, quality and information value of available principal study; 4) Data analysis: Including data reduced, illustrated, contrasted as well as conclusion; 5) Presenting results: Interpretation of evidence and presentation of findings [6] [7].

\subsection{Search Strategy}

For this review, PICo (Participant, area of Interest, Context) was used to search data, in which Participant (P) referred to nurses, area of Interest (I) consisted of factors, and Context (Co) was the end of life care. The first step was to determine 
the research question, namely: What factors are relating to nurses' end-of-life care? Articles for this review were selected from databases such as Google Scholar, PubMed, SCOPUS, ProQuest and Web of Science, with keywords which were identified by PICo: "nurse" AND "end of life care" ("dying care" OR "death care", OR "near end stage of life care" OR "palliative care" OR "hospice care" OR "near death" OR "quality of dying patients care"), AND "nurses". Inclusion criteria considered were "English and Chinese language publication, studies of end of life care of nurses, publication time was 2010 to 2019." Exclusion criteria were "Studies of end-of-life care about children." The initial search from the databases yielded 260 articles. After having filtered these articles based on inclusion criteria, fifteen full text articles were selected for further analysis and synthesis of the factors relating to nurses' end of life care.

\subsection{Data Analysis}

At first, using a table, key details of studies were identified. These details included authors, title, publication year, aim, type of study, sample, tools, findings, critique strength and weakness of the studies. By reading the fifteen selected articles several times, the first author focused on finding sections, and these were then reviewed by the second author to try to discover sections of the findings that answered the research question. The author categorized every finding in the matrix table of factors influencing nurses' end-of-life care to find similarities and differences in the findings and come up with a synthesis regarding these findings. Two synthesized findings emerged as Nurses' demographic factors and Modifiable factors. During the process of data synthesis, the author maintained the important findings of major studies. Meanwhile, for every paper a description was given as to how data were collected and processed, also showing their research methods, which were then written into the extracted table.

\section{Results}

The findings were based on fifteen articles which consisted of one qualitative study and thirteen cross-sectional studies/correlation studies and one mixed method-study. Most studies used survey questionnaires via mail while only one study used an online survey method to collect data. Regarding setting of the study, results showed that four studies explored end-of-life care in governmental hospitals and one study conducted a survey in a non-governmental hospital. Furthermore, two studies came from university hospitals, whereas a non-university hospital, public institutions, a teaching hospital and a non-teaching hospital, an intermountain region hospital and sites of the supply of tranquility care such as rest homes and homes for the elderly, all constituted the setting for only one study respectively. There was no study conducted in community hospitals. These studies were conducted in various areas (Taiwan of China, Thailand, USA, Czech Republic, Ireland, Iran, Ethiopia, Italy and Chile). The main departments for data collection were oncology, emergency ward, internal medicine and sur- 
gery general ward, clinic units and ICU. Synthesis of the findings established two categories of factors relating to nurses' end-of-life care, namely nurses' demographic factors and modifiable factors.

\subsection{Nurses' Demographic Factors}

The delivery of end-of-life care was undoubtedly influenced by many nurses' demographic factors. These factors included their age, work experience, educational level and experience of the death of family members/friends which could affect their own ability to care.

\subsubsection{Age}

At different ages, nurses have different world views including views on death. Of course, at the same time and because they have little experience contacting with the terminally ill, which will cause different behaviors. A correlational study showed that a factor for high application frequency of the activity series enhancing physical comfort for dying patients was nurses' age. For younger nurses, it was very difficult to provide care for the dying because of their fear of death [8]. Older nurses can offer more confidence during the period of care in managing dying patients 'symptoms than younger nurses $(Z=5.80, p<0.01)$ [9]. The younger nurses were inclined to report a higher level of fearing death than older nurses who had a positive effect on their practice of end-of-life care $(r=0.075, p$ $<0.05)[10]$.

\subsubsection{Years of Working Experience}

End-of-life care concentrated on providing comfortable care for patients, which needed a high-level or expert practice. One of elements relating to a nurse's caring action for the dying sick person was working experience. A study conducted in the USA reported that working years of caring experience were positively correlated to comfortable standard of nurses' actions of end-of-life care (F $(9,131.57)=2.22, p<0.05)[11]$. Another study also showed that nurses with few years of working experience had significantly lower levels of being comfortable than those with many years. If nurses had more professional experience of nursing dying patients, they would have more confidence in relieving symptoms of terminal stage patients $(\mathrm{Z}=7.46, p<0.01)[9]$.

\subsubsection{Level of Education}

As nurses became conscious of playing a major role in dying patient's care, they saw the value of giving more importance to integrating the care content in nursing education [12]. The level of education had been found to show significant statistical correlation with practice of nurses' end of life care $(p<0.03)$. The nurses who had a master degree, were observed to be better at end-of-life care [13] [14].

\subsubsection{Experience of the Death of Family/Friend}

If nurses have more experience of the death of family members/friends, they 
could then provide more targeted messages, suggestions, and high-level nursing care service for dying patients, because they could assist patients in getting favorable and all-around care. A study conducted in Taiwan of China revealed a major predictor that nurses who underwent the experience of death of family members or friends could get a higher score in service of end-of-life care ( $\mathrm{F}=$ $5.3, p<0.001)[15]$.

\subsection{Modifiable Factors}

As for modifiable factors, these played a significant role in the process of perfecting the practical ability of nurses. They all constituted the basis of the quality of nurse service. Enough knowledge, attitude towards death, enhancing confidence, good relationships, environment and resources of care, communication with each other, nursing activities, philosophy and culture in organization of care, high level skills and end-of-life care training could be given attention by institutions responsible for some policies, efforts and opportunities, which would improve care quality of nurses for patients nearing death.

\subsubsection{Knowledge toward End-of-Life Care}

Positive correlation between nurses' practice and knowledge towards end-of-life-care was signified in several studies [1] [14] [16]. Nurses learned more plentiful knowledge of end-of-life care, and they engendered better favorable practice in caring for dying patients $(r=0.57, p<0.01)$, which revealed a strong correlation [14]. In addition, nurses who had good knowledge had a favorable and positive attitude of end-of-life care which could strengthen confidence of nurses in nursing dying patients $(p<0.02)$, so it also showed indirectly that there is a correlation between knowledge and practice [1]. For example, nurses' knowledge of advanced directives had positive relationship with their confidence perceived in the end-of-life care [9]. In addition, another article described that a predictor relating nurses' end-of-life care was the knowledge/expertise of health care professionals which showed an active correlation with the ability to provide a comfortable death at end of life $(p<0.05)$ [16].

\subsubsection{Attitude towards Death}

The attitude of nurses and the quality of nursing practices are mutually reinforcing. There was a study conducted in Taiwan showing that the healthcare staff's attitudes about caring for dying patients were strongly positively correlated with their practices $(r=0.42, p<0.01)$ [14]. Another study conducted in Jordan's capital Amman also described how nurses' attitudes towards death could be significantly associated with nursing practice for patients near death ( $p$ $<0.003$ ) [8]. In the usual work of nurses, we can find that patients care about nurses' attitude towards them. During the period of illness, patients' self-esteem becomes very strong, so their sensitivity to nurses' attitude will increase.

\subsubsection{Confidence in the End-of-Life Care}

Confidence in end-of-life care of nurses is a factor relating to their practice's 
competency and quality of care. This is shown in the study of Coffey et al. (2016) that surveyed nurses who came from five areas including Hong Kong of China, Ireland, Israel, US and Italy. This study found that there was a significant positive correlation between both confidence of dealing with sick persons and comfort at the last stage of life (Rs $=0.671, p<0.01$ ) [9]. Nurses being short of confidence and competence might be an obstacle in being able to supply a high-level quality of care for patients who were near death. Nurses who felt more confident could provide more comfortable care at the patients' end of life [9].

\subsubsection{Relationship}

Relationship means relationship within the healthcare team and relationship between nurse and patients. Nurses noted that closely aligned interdisciplinary teams of end-of-life care which included doctors, nurses, social staff and administrators could minimize the time and facilitate transfers to more suitable settings for terminal patients near death [12]. In addition, nurses viewed that "understanding the patient" and "producing an intimate relation" was a sticking point and a determinative standard of peaceful care [17], while at the same time constituting a convenient way to find problems of patients.

\subsubsection{Environment and Resources}

The nurses working in different units, regional areas and countries had different levels of confidence, comfortable care, knowledge, and experience toward palliative care [1] [9] [12] [13]. A study showed that the nurses who worked in intensive and emergency departments $(p<0.001)$ and who had gone through the process of nursing terminal patients had better practice of end-of-life care [13]. Another article described that nurses who worked in oncology departments showed a higher level of being comfortable than nurses in other wards (ICU/ Telemetry) towards dying patients' care $(\mathrm{F}(6,110)=2.49, p<0.05)[11]$. At the same time, a study also identified that nurses belonging to medical wards (AOR $=2.751 ; 95 \% \mathrm{CI} 1.223-6.188 ; p=0.003)$ and surgical wards $(\mathrm{AOR}=3.445 \mathrm{CI}$ $1.479-4.399 ; p=0.003$ ) had better positive attitudes towards end-of-life care [4]. In addition, an important issue in end-of-life care was the physical condition and adequate supplies that could assure secrecy and honor of the patients and their family. Meanwhile, adequate resources could provide a better ability of symptom management for patients near death stage [17].

Many studies also argued that the nurses' work environment of hospice care facilities was an important predictor of nurses supporting desirable care and therapy for patients at the end of life stage $(72.1 \%$ vs. $67.5 \%, p \leq 0.01)$ [15]. It was also a mainly predictive factor for the effectively operating rate of activities referring to physical comfort $(\mathrm{OR}=10.40 ; 95 \%, \mathrm{CI}=3.17-34.10 ; p=0.0001)$ [10]. The working environment constituted one of the foundations for quality of nursing care. Nurses believed that nurses, patients and their families needed to be provided some resources such as ample private space and time, and suitable medical staff from interdisciplinary fields [12]. The author also found a study 
which described how with a respectful structure of care, the coordination and consistency with care frequency was associated with a better level of quality during the dying process $(p<0.05)[18]$.

\subsubsection{Communication}

Available intervening elements which could enhance the level of end-of-life care could include discussions between doctors and nurses, for example, during the period of end-of-life discussions, conversation topics between doctors and nurses had a relationship with higher levels of peaceful care. These topics consisted of family questions $(\beta=0.97, p<0.001)$, family dynamics $(\beta=0.53, \mathrm{p}=$ $0.003)$, spiritual/religious issues $(\beta=0.90, p<0.001)$, cultural issues $(\beta=1.09, p$ $<0.001)$ and nurses' consideration related to sick persons or their families $(\beta=$ $-0.60, p=0.001$ ), which had a positive correlation with higher-level practice of nursing within the last stage of patients' life [19].

\subsubsection{Nursing Activities}

Comfortable actions of promotion played an essential role in the process of caring for dying patients. Any activity that offered psychological comfort would be one of the most significant links in the aspect of patient support. Activities which included monitoring pain, basial care, and other promotion all could support physical comfort for ill patients at the end of life stage. The frequencies of three activities involving biological $(r=-0.22, p<0.01)$, psychological $(r=$ $-0.21, p<0.01)$, and spiritual $(r=-0.21, p<0.01)$ dimensionality had a negative correlation with the frequency of supplying care to patients at end of life [3].

\subsubsection{Philosophy and Culture in Organization of Care}

The factor that focused on elements of philosophy which emphasized comfort and dignity could influence nurses' end-of-life care [17]. A harmonious kind of culture of nursing organizations included harmonious interpersonal relationships, harmonious nursing relationships, harmonious medical relationships [20] and the sharing of knowledge and experience, constituting a platform that was often a key factor in promoting the quality of care.

\subsubsection{Skills}

For qualified nurses, whose technical requirements are very high, they may have a different technical skills level, so delivering a comfortable standard of care for terminal patients would not be the same. A study reported that nurses who had a high-level of comfortable care in terms of technical and logistic aspects could provide more comprehensive and holistic nursing care, partially, when looked at as a performance of nurses' presentation and capability to administrate in the whole process of dying [12]. Another study showed that the physical symptoms control such as control of complication and pain was connected with the level of dying quality $(p<0.05)$. Its findings supported the view that controlling symptoms would depend on the skills of care which came from physicians and nurses, who might offer what was needed to reach a better level of quality of care for 
terminal patients [18].

\subsubsection{End of Life Care Training}

Training in the field of end-of-life care is a major element enhancing confidence and ability of nurses' end-of-life care. A study also reported that a significant positive correlation exists between sufficient training of end-of-life care and both comfort and nurses' self-confidence $(r=0.533, p<0.01)$ in dealing with patients' symptoms $(r=0.47, p<0.01)$ at end stage of life [9].

\section{Discussion}

\subsection{Nurses' Demographic Factors}

Nurses' demographic factors include age, years of working experience, level of education and experience of the death of family/friend. In fact, these four factors interact. Young nurses have a lack of work experience and experience fear of death, and if they are also resistant, then nurses' own education and training are common conditions that limit their work experience. As training in education levels and related expertise deepens, young nurses who can get experience will improve their practice level of end of life care. For example, there are some signs which, although less obvious, might be detectable by experienced nurses, such as withdrawal, refusing food or drink and consistent requests to see family members and loved ones [21]. Although the importance of nurses' education as a means of preparing them in dealing with patient death and dying has been highlighted, different gaps still existed in aspect of educational training related to palliative care in China.

\subsection{Modifiable Factors}

Modifiable factors include knowledge, attitude, confidence, relationship, environment and resources, communication, activities, philosophy and culture in organization of care, skills and training.

As for nurses, end-of-life care knowledge and their practice cannot be separable, because good knowledge can guide nurses to show good practice and confidence towards end-of-life care. Some studies showed that for nurses, being short of knowledge and educational material was a public point in the care of end of life, so they would be demanded to accumulate information about end-of-life care by studying and training [21] [22]. Research also reported that some information of end-of-life care was gained by nurses who communicated with terminal patients, family members and healthcare team and took part in related educational programs. Moreover, in order to enhance nursing practice for dying patients, nurses must possess numerous skills to handle problems [16]. For example, they need to have good skills and knowledge in dealing with interpersonal trouble between patients and their family when they have to make decisions [22] [23].

In fact, nurses also play a key role in the process of communication of all involved in caring. Nurses should be a bridge between doctors and patients. If 
communication topics are close to what patients and their families are eager to know, they can help them build good relationships, and symptom management will be done well which mainly depends on coordinating with the health team (physicians and nurses) [18]. For patients with advanced levels of illness, pain relief is their most desired. The end-of-life care theory also included a point about being free from pain, so nurses are demanded to cooperate with doctors to provide measures for patients to reduce pain and control symptoms, because pain symptom would influence patient's psychological, physical, and spiritual health [20]. Of course, these were also based on nurses' professional competence to offer higher level practice of end-of-life care for ill clients and their family members [16].

Nurses usually had sentimental feelings towards patients during the working process. They said that they repressed or concealed their feelings for a long time when they faced patients. However, these emotions can influence the nurses' ability and attitude towards caring for dying patients [12]. Moreover, two studies [8] [14] have shown that the nurse's attitude was an important factor related to nurses' practice, as a positive attitude of nurses could enable patients to get comfortable care at the stage nearing death of patients. Therefore, nurse's attitude is a major influencing element.

Finally, the environment and resources in which nurses are located are also important because it is the basis of nursing practice. There is a saying in China: "For the clever woman it is difficult to cook without rice." It is essential, that's what it means. This is because the environment and resources can provide nurses with enough time, space and opportunities for learning and training. Moreover, it can protect patients' privacy and improve the comfort of patients and their families. Limited environment and resources do not enable nurses to fully develop their abilities of end-of-life care [12].

\section{Conclusion}

This integrative review identified many factors related to nurses' end-of-life care. These factors included nurses' demographic factors and modifiable factors. Factors that were identified as having moderate to strong correlation with nurses' end-of-life care are: knowledge toward end-of-life care, level of education and training of end-of-life care, attitude toward death, environment and resources as well as communication topics. Predictive factors to nurses' peaceful care were the working environment of nurses, and the care facilities, as well as experience of death of family member/friend. However, the studies were limited to teaching hospitals and hospice institutions. Two studies involved surveys conducted in China while a rare study was done in a community hospital.

\section{Recommendations}

\subsection{Nursing Practice}

The results of this literature review can be used to formulate suggestions to the 
hospitals, especially when it comes to modifiable factors. Nurse administrators in the hospitals should consider modifiable factors in improving or enhancing nurses' practice of comfortable care. Nurses working in clinical practice should have a high level of knowledge in tranquility care and a positive point of view towards death to improve their level of practice in dying patients care. In view of the fact that the philosophy and culture in an organization could influence the practice of nurses' care, nurses should constantly improve their knowledge in practice. In addition, the state of the facilities of the nurses' working environment is also very important. By providing a good working environment and advanced facilities, health institutions can enable nurses to better deliver comfortable services for dying patients. Therefore, as head of hospitals, they need to pay attention to these factors and improve them to assure that nurses are supplied with what they need for their practice.

\subsection{Nursing Research}

The factors identified in this literature review can be useful in formulating recommendations for future nursing research. In addition, the next step of research would have to involve exploring the end-of-life care facilities which constitute a significant predictor relating to nurses' end-of-life care to enhance nurses' ability in getting rid of passive influences in the course of events of caring for sick clients and their family at the end of life stage. Study methods could also be more diversified, which could combine quantitative research with qualitative research in order to go deeper into studying the factors relating to nurses' end-of-life care. Community hospitals should be of interest as setting in a future study.

\section{Conflicts of Interest}

The authors declare no conflicts of interest regarding the publication of this paper.

\section{References}

[1] Abate, T.A., Amdie, Z.F., Bayu, H.N., Gebeyehu, D. and Marianm, T. (2019) Knowledge, Attitude and Associated Factors towards End of Life Care among Nurses Working in Amhara Referral Hospitals, Northwest Ethiopia. BMC Research Notes, 12, 521. https://doi.org/10.1186/s13104-019-4567-7

[2] Ranse, K., Yates, P. and Coyer, F. (2012) End-of-Life Care in the Intensive Care Setting: A Descriptive Exploratory Qualitative Study of Nurses' Beliefs and Practices. Australian Critical Care, 25, 4-12. https://doi.org/10.1016/j.aucc.2011.04.004

[3] Kisvetrova, H., Koloudík, S.D., Joanovič, E., Konečna, J. and Mikšova, Z. (2015) Dying Care Interventions in the Intensive Care Unit. Journal of Nursing Scholarship, 48, 139-146. https://doi.org/10.1111/jnu.12191

[4] Kassa, H., Murugan, R., Zewdu, F., Hailu, M. and Woldeyohannes, D. (2014) Assessment of Knowledge, Attitude and Practice and Associated Factors towards Palliative Care among Nurses Working in Selected Hospitals, Addis Ababa, Ethiopia. BMC Palliative Care, 13, 6. https://doi.org/10.1186/1472-684x-13-6 
[5] China Health Committee (2019) Push the Peace and Care across the Country as Soon as Possible. http://news.sina.com.cn/c/2019-06-10/doc-ihvhiqay4641974.shtml.

[6] Hopia, H., Latvala, E. and Liimatainen, L. (2016) Reviewing the Methodology of Integrative Review. Journal of the Caring Sciences, 30, 662-669.

https://doi.org/10.1111/scs.12327

[7] Whittemore, R. and Knafl, K. (2005) The Integrative Review: Updated Methodology. Journal of Advanced Nursing, 52, 546-553. https://doi.org/10.1111/j.1365-2648.2005.03621.x

[8] Abu Hasheesh, O.M., Abo Zeid, L.S., El-Said, G.S. and Alhujaili, D.A. (2013) Nurses' Characteristics and Their Attitudes toward Death and Caring for Dying Patients in a Public Hospital in Jordan. Health Science Journal, 7, 384-394.

[9] Coffey, A., McCarthy, M.G., Weathers, E., Friedman. I.M., Gallo, K., Ehrenfeld, M., Chan, S., William, H.C., Poletti, P., Zanotti, R., Molloy, W.D., McGlade, C., Fitzpatrick, J.J. and Itzhaki, M. (2016) Nurses' Knowledge of Advance Directives and Perceived Confidence in End-of-Life Care: A Cross Sectional Study in Five Countries. International Journal of Nursing Practice, 22, 247-257. https://doi.org/10.1111/ijn.12417

[10] Kisvetrova, H., Vodova, V.S. and Kloud'ık, S.D. (2017) Comfort-Supporting Nursing Activities for End-of-Life Patients in an Institutionalized Environment. Journal of Nursing Scholarship, 50, 126-133. https://doi.org/10.1111/jnu.12341

[11] Moir, C., Roberts, R., Martz, K., Perry, J. and Tivis, J.L. (2015) Communicating with Patients and Their Families about Palliative and End of Life: Comfort and Educational Needs of Staff RNs. International Journal Palliative Nursing, 21, 109-112. https://doi.org/10.12968/ijpn.2015.21.3.109

[12] Wolf, A.L., Delao, M.A., Perhats, C., Clark, R.P., Moon, D.M., Baker, M.K. and Lenehan, G. (2015) Exploring the Management of Death: Emergency Nurses' Perceptions of Challenges and Facilitators in the Provision of End-of-Life Care in the Emergency Department. Journal of Emergency Nursing, 41, e23-e33. https://doi.org/10.1016/j.jen.2015.05.018

[13] Farmani, H.A., Mirhfez, R.S., Kavosi, A., Pasha, M.A., Nasab, J.A., Mohammadi, G., Moeini, V., Aryayi Far, M.R. and Movahedi, A. (2018) Dataset on the Nurses' Knowledge, Attitude and Practice towards Palliative Care. Data in Brief, 22, 319-325. https://doi.org/10.1016/j.dib.2018.11.133

[14] Huang, L.C., Tung, H.J. and Lin, P.C. (2019) Associations among Knowledge, Attitudes, and Practices toward Palliative Care Consultation Service in Healthcare Staffs: A Cross-Sectional Study. Plos One, 14, e0223754. https://doi.org/10.1371/journal.pone.0223754

[15] Lee, K.A., Brennan, W.C., Meterko, M. and Ersek, M. (2015) Organization of Nursing and Quality of Care for Veterans at the End of Life. Journal Pain Symptom Manage, 49, 570-577. https://doi.org/10.1016/j.jpainsymman.2014.07.002

[16] Cagle, G.J., Pek, J., Clifford, M., Guralnik, J. and Zimmerman, S. (2015) Correlates of a Good Death and the Impact of Hospice Involvement: Findings from the $\mathrm{Na}$ tional Survey of Households Affected by Cancer. Support Care Cancer, 23, 809-818. https://doi.org/10.1007/s00520-014-2404-Z

[17] Casey, D., Murphy, K., Leime, N.A., Larkin, P., Payne, S., Froggatt, A.K. and Shea, O.E. (2010) Dying Well: Factors That Influence the Provision of Good End-of-Life Care for Older People in Acute and Long-Stay Care Settings in Ireland. Journal of Clinical Nursing, 20, 1824-1833. https://doi.org/10.1111/j.1365-2702.2010.03628.x

[18] Venegas, E.M. and Alvarado, S.O. (2010) Factors Related to the Quality of the Dy- 
ing Process in Cancer Patients. Revista Latino-Americana de Enfermagem, 18, 725-731. https://www.eerp.usp.br/rlae https://doi.org/10.1590/s0104-11692010000400010

[19] Ramos, J.K., Downey, L., Nielsen, L.E., Treece, D.P., Shannon, E.S., Curtis, R.J. and Endelberg, A.R. (2015) Using Nurse Ratings of Physician Communication in the ICU to Identify Potential Targets for Interventions to Improve End-of-Life Care. Journal of Palliative Medicine, 19, 292-299. https://doi.org/10.1089/jpm.2015.0155

[20] Marshall, K.V. and Given, A.B. (2018) Factors Associated with Medication Beliefs in Patients with Cancer: An Integrative Review. Oncology Nursing Forum, 45, 508-526. https://doi.org/10.1188/18.onf.508-526

[21] Fitch, M.I., Dasgupta, T. and Ford, B. (2016) Achieving Excellence in Palliative Care: Perspectives of Health Care Professionals. Asia-Pacific Journal of Oncology Nursing, 3, 66-72. https://doi.org/10.4103/2347-5625.164999

[22] McCourt, R., Power, J.J. and Glackin, M. (2013) General Nurses' Experiences of End-of-Life Care in the Acute Hospital Setting: A Literature Reviews. International Journal of Palliative Nursing, 19, 510-516.

https://doi.org/10.12968/ijpn.2013.19.10.510

[23] Prompahakul, C., Nilmanat, K. and Kongsuwan, W. (2011) Review: Factors Relating to Nurses' Caring Behaviors for Dying Patients. Nurse Media Journal of Nursing, 1, 15-27. 